\title{
A Note on the Stochastic Comparison in Production Yield Management
}

\author{
Kyungchul Park ${ }^{\dagger}$ \\ School of Business, Myongji University \\ 생산 수율 관리 문제와 확률적 비교 \\ 박 경 철 \\ 명지대학교 경영대학 경영학과

\begin{abstract}
The single-period production inventory control problem under random yield is considered to analyze the impact of the yield characteristics on the firm's profit. We use the stochastic comparison as a main vehicle to compare the profits resulted under different random yields. Commonly used stochastic orderings are addressed with an analysis of their implications on the firm's profit. Moreover, a distribution-free bound on the profit is derived.
\end{abstract}

Keywords: Random Yield, Newsvendor Problem, Stochastic Orders

\section{Introduction}

Random yields are so pervasive in many production environments. Production firms make their effort to improve the yield quality with the goal of achieving more profit. In many cases, the yield quality improvement aims at reducing the variability of the final yield. Moreover, making random yield stochastically larger (hence increasing the possibility of obtaining good units) has been advocated as an effective way to improve the firm's profitability.

However, there are circumstances that stochastically large random yields may harm the long-term profit of the producers. Gupta and Cooper (2005) gave an example of such counterintuitive phenomena. They also studied various cases where the increase in variance of the yield will be desirable. This note extends their results in different perspectives. Specifically, we focus on the various stochastic orderings and their implications on the firm's profit. To this end, we will consider the single-period problem (newsvendor problem) with ran- dom yield but with certain demand. In many industries, the assumption of certain demand is not so unrealistic, as pointed out by Keren (2009). Moreover, focusing solely on the random yield can make it easier to illuminate the important issues related to yield quality management.

After presenting some preliminary results, we will consider various stochastic orders including the usual stochastic order and the convex order. Assuming two random yields satisfy the specified stochastic order relation, the corresponding profits will be compared. Moreover, we will present a distribution-free bound on the profit. The results can be used to give an insight into the real benefits of the firm's product/process quality improvement initiatives.

Previous researches on the random yield include Gerchak et al. (1986), Gerchak and Parlar (1990), Henig and Gerchak (1990), Anupindi and Akella (1993), to name a few. Yano and Lee (1995) gave a comprehensive review on the random yields under the lot sizing contexts. Khouja (1999) serves as a good reference on the newsvendor problem.

This work was supported by 2008 Research Fund of Myongji University.

† Corresponding author : Professor Kyungchul Park, School of Business, Myongji University, 50-3 Namgajwa-dong, Seodaemun-gu, Seoul, 120-728 Korea, Tel : +82-2-300-7181, Fax :+82-2-300-0734, E-mail : daneel@mju.ac.kr

Received June 3, 2014; Accepted June 11, 2014. 


\section{Model Description and Preliminary Results}

\subsection{Inventory Control Problem under Random Yield}

Let $r, c, h$, and $p$ denote the retail price, the unit production cost, the unit holding (or disposal) cost, and the unit penalty cost, respectively. The values of $r, c$, and $p$ are assumed to be nonnegative. However, $h$ can take the negative value representing the salvage value. The number of good units obtained from the production is a random variable and is denoted as $U x$, where $x$ is a production lot size and $U$ is a nonnegative random variable (yield). Let $F_{U}$ be its distribution function. We assume that $(r+p) \mathrm{E} U>c$ for the production to be beneficial. In addition, to prevent stocking infinite inventory, it is assumed $c+h \mathrm{E} U>0$. Let $\lambda$ be a known demand. We write $U={ }_{s t} V$ for two random variables $U$ and $V$ if they have the same distribution functions.

Suppose the realized yield is $\xi$, then the profit is written as follows:

$$
\begin{aligned}
\pi_{U}(x, \xi, \lambda)= & (r+h) \lambda \\
& -(c+h \xi) x-(r+h+p)(\lambda-\xi x)^{+} .
\end{aligned}
$$

where $a^{+}=\max (a, 0)$ for all real numbers $a$.

The expected profit to be maximized is

$$
\begin{aligned}
\Pi_{U}(x, \lambda)= & \mathrm{E} \pi_{U}(x, U, \lambda)=(r+h) \lambda \\
& -(c+h \mathrm{E} U) x-(r+h+p) \mathrm{E}(\lambda-U x)^{+} .
\end{aligned}
$$

Dividing (2) by $\lambda$ results in

$$
\begin{aligned}
\Pi_{U}(x / \lambda, 1)= & (r+h)-(c+h \mathrm{E} U)(x / \lambda) \\
& -(r+h+p) \mathrm{E}(1-U(x / \lambda))^{+} .
\end{aligned}
$$

Thus we have $\Pi(x, \lambda)=\lambda \Pi(x / \lambda, 1)$, and so without loss of generality, we can assume the demand equals 1 , which is assumed from now.

The expected profit can be rewritten as follows :

$$
\begin{aligned}
\Pi_{U}(x)= & \mathrm{E} \pi_{U}(x, U)=(r+h)-(c+h \mathrm{E} U) x \\
& -(r+h+p) \mathrm{E}(1-U x)^{+}
\end{aligned}
$$

The optimal lot size and the maximum expected profit are given in the following Theorem 1 (for details, see Park and Lee, 2014).

Theorem 1 : Let $U$ be a continuous nonnegative random variable.

1. The optimal lot size is $x^{*}=1 / \alpha>0$ where $\alpha$ is given by

$$
\int_{0}^{\alpha} \xi d F_{U}(\xi)=(c+h \mathrm{E} U) /(r+h+p) .
$$

2. The maximum expected profit is

$$
\Pi_{U}^{*}=\Pi_{U}\left(x^{*}\right)=\Pi_{U}(1 / \alpha)=(r+h)-(r+h+p) F_{U}(\alpha) .(6)
$$

Note that the optimality condition (5) is well-defined since $(c+h \mathrm{E} U) /(r+h+p)<\mathrm{E} U$.

\subsection{Stochastic Ordering}

A stochastic order is used to define a partial order among the random variables. Shaked and Shanthikumar (1994) and Muller and Stoyan (2002) present comprehensive background. In this note, we consider the integral stochastic orders defined as follows.

Definition $1:$ Let $\mathfrak{I}$ be a nonempty class of functions. For two random variables $X$ and $Y, X \leq_{\jmath} Y$ holds if and only if $\mathrm{E} f(X) \leq \mathrm{E} f(Y)$ for all $f \in \mathfrak{I}$.

1. If $\mathfrak{I}$ is the class of increasing functions, then the order is called a usual stochastic order and is denoted as $\leq_{s t}$.

2. If $\mathfrak{I}$ is the class of convex functions, then the order is called a convex order and is denoted as $\leq$.

3. If $\mathfrak{I}$ is the class of increasing concave functions, then the order is called an increasing concave order and is denoted as $\leq_{i c v}$.

It is well-known that given two random variables $X$ and $Y$, $Y$ is stochastically larger than $X$ (that is, $X \leq_{s t} Y$ ) if and only if

$$
F_{X}(t) \geq F_{Y}(t) \text { for all real numbers } t .
$$

Briefly, $X \leq_{s t} Y$ implies that it is more likely that the random variable $Y$ takes larger values than $X$. Hence it may seem to be intuitively clear that a stochastically larger yield results in more profit. However, as Gupta and Cooper (2005) have shown, it is possible that a stochastically larger yield can result in less profit.

For two nonnegative random variables $X$ and $Y, X \leq{ }_{s t} Y$ implies $\mathrm{E} X \leq \mathrm{E} Y$. If $X \leq{ }_{s t} Y$ and $\mathrm{E} X \leq \mathrm{E} Y$, then $X={ }_{s t} Y$ (Muller and Stoyan 2002). Hence the usual stochastic order is not helpful when investigating the effect of variance reduction in the random yield. The convex order is in order in this case, since $X \leq_{c x} Y$ implies $\mathrm{E} X=\mathrm{E} Y$ and $\operatorname{Var}(X) \leq \operatorname{Var}(Y)$.

\section{Main Results}

This section explores the implications of the various stochastic orders on the firm's profit. We will consider the usual sto- 
chastic order, the convex order, and the increasing concave order. Based on the results, the mean-preserving transform will be analyzed and a distribution-free bound on the profit will also be derived.

\subsection{Usual Stochastic Order and Increasing Concave Order}

Let two nonnegative random yields $U$ and $V$ be given and they satisfy $U \leq{ }_{s t} V$. As mentioned in the previous section, it does not imply in general $\Pi_{U}^{*} \leq \Pi_{V}^{*}$. However, a sufficient condition for the relation to hold can be found by investigating the Theorem 1.

Proposition 1 : If $U \leq{ }_{s t} V$ and $x^{*} \leq y^{*}$ (the corresponding optimal lot sizes), then $\Pi_{U}^{*} \leq \Pi_{V}^{*}$.

Proof : Note that $F_{V}\left(1 / y^{*}\right) \leq F_{U}\left(1 / y^{*}\right) \leq F_{U}\left(1 / x^{*}\right)$. So by Theorem 1 , the result follows.

Though in general, $U \leq{ }_{s t} V$ does not imply $\Pi_{U}^{*} \leq \Pi_{V}^{*}$, it is true when $h \leq 0$. In this case, the profit function (1) is increasing in $\xi$. Hence we get the following result.

Proposition 2 : Let $h \leq 0$. Then $U \leq{ }_{s t} V$ imply $\Pi_{U} \geq \Pi_{V}$ for all $x \geq 0$, hence $\Pi_{U}^{*} \leq \Pi_{V}^{*}$.

The above proposition tells that if a leftover unit has a nonnegative salvage value, then the stochastically larger yield always gives more profit. Hence the previously mentioned counter-intuitive case occurs only when positive holding costs arise.

Note that the Proposition 2 also holds when $U \leq_{i c t} V$ since the profit function is an increasing concave function in $\xi$. Since $U \leq{ }_{s t} V$ implies $U \leq{ }_{i c t} V$, the proposition with the increased concave order is more general.

\subsection{Convex Order}

Let two nonnegative random yields $U$ and $V$ be given with $U \leq{ }_{c x} V$. Let us define

$$
\begin{aligned}
\phi_{U}(x, \xi) & =(r+h)-\pi_{U}(x, \xi) \\
& =(c+h \xi) x+(r+h+p)(1-\xi x)^{+}
\end{aligned}
$$

Also let $\Phi_{U}(x)=\mathrm{E} \phi_{U}(x, U)$. It is clear that $\Pi_{U} \geq \Pi_{V}$ if and only if $\Phi_{U}(x) \leq \Phi_{V}(x)$.

Note that for each nonnegative number $x$, the function $\phi_{U}$ is a convex function in $\xi$. Hence $U \leq_{c x} V$ implies $\Pi_{U}(x) \geq$ $\Pi_{V}(x)$ for all nonnegative numbers $x$ (which further implies that $\left.\Pi_{U}^{*} \geq \Pi_{V}^{*}\right)$. The following proposition states that the converse also holds.
Proposition 3 : Let $\mathrm{E} U=\mathrm{E} V$. Then $\Pi_{U}(x) \geq \Pi_{V}(x)$ holds for all $x \geq 0$ if and only if $U \leq{ }_{c x} V$.

Proof : We only need to prove the "only if" part. $\Pi_{U}(x) \geq$ $\Pi_{V}(x)$ is equivalent to $\Phi_{U}(x) \leq \Phi_{V}(x)$, which further is equivalent to $\mathrm{E}(1-U x)^{+} \leq \mathrm{E}(1-V x)^{+}$for all $x \geq 0$. The relation can be restated as $\mathrm{E}(t-U)^{+} \leq \mathrm{E}(t-V)^{+}$for all $t$ by noting that $U$ and $V$ are nonnegative random variables. Since $\mathrm{E}(t-U)^{+}=(t-\mathrm{E} U)+\mathrm{E}(U-t)^{+}$, the relation implies $\mathrm{E}(U$ $-t)^{+} \leq \mathrm{E}(V-t)^{+}$for all $t$, which means that $U \leq{ }_{c x} V$.

The above proposition says that if $U \leq_{c x} V$, then for any production lot size, the profit under the random yield $U$ is not smaller than that under $V$. In Muller and Stoyan (2002), the comparison criteria for commonly used parametric distributions can be found. For many kinds of distributions used to model the random yield such as the (truncated) normal distribution and the lognormal distribution, the condition $U \leq_{c x} V$ is equivalent to $\operatorname{Var}(U) \leq \operatorname{Var}(V)$. Thus if the random yield can be shown to follow such a distribution, the variance reduction (while preserving the mean) always results in improved profit. Moreover, even if the same lot size is used (for instance, due to some technical reasons), the profit should be improved. Hence in many cases, the process quality improvement resulting in smaller variance leads to more profit.

\subsection{Mean-Preserving Transform}

As an application of the Proposition 3, let us consider the case of mean-preserving transform (Gerchak and Mossman 1992). Specifically, let us consider the family of random yields $\{U(a)\}$ defined as $U(a)=(1-a) U+a \mu$, where $U$ is a nonnegative random variable, $\mu=\mathrm{E} U$ and $0 \leq a \leq 1$. Since $\mathrm{E} U(a)=\mu$ and $\operatorname{Var}[U(a)]=(1-a)^{2} \operatorname{Var}[U]$, the mean is fixed but the variance is decreasing in $a$ (so the name meanpreserving transform is used). By noting that $\operatorname{Pr}[U(a) \leq t]=$ $\operatorname{Pr}[U \leq(t-a \mu) /(1-a)]$, we have $F_{U(a)}(t)=F_{U}((t-a \mu) /$ $(1-a))$. In the following, we will show that if $o<a<b<1$, then $U(b) \leq{ }_{c x} U(a)$, which implies the variance-reduction results in the increased profit. To this end, we need the following lemma.

Lemma 1 : (Muller and Stoyan 2002) Let $X$ and $Y$ be random variables with the same mean. If there is some $t_{0}$ such that $F_{x}(t) \leq F_{Y}(t)$ for all $t \leq t_{0}$ and $F_{x}(t) \geq F_{Y}(t)$ for all $t>t_{0}$, then $X \leq{ }_{c x} Y$.

The condition given in Lemma 1 is called as a cut criterion.

Proposition 4 : Let $U(a)=(1-a) U+a \mu$ where $0 \leq a \leq 1$. Then $\Pi^{*}(a)$ is increasing in $a$.

Proof : Let $0<a<b<1$. Then $F_{U(a)}(t) \geq F_{U(b)}(t)$ if and only if $t \leq \mu$. Hence $U(a)$ and $U(b)$ satisfy the cut criterion and so $U(b) \leq{ }_{c x} U(a)$. Then the result follows from the Pro- 
position 3 .

\subsection{Distribution-Free Bound on the Maximum Profit}

Let us define $M_{\mu}^{[a, b]}=\{U \mid \mathrm{E} U=\mu, \operatorname{Pr}[a \leq U \leq b]=1\}$, that is, the set of random variables with the same mean and with the common support $[a, b]$, where $0 \leq a<b$. Consider the order $\leq_{c x}$ defined on $M_{\mu}^{[a, b]}$. Let $\underline{X}$ and $\bar{X}$ be the minimum and the maximum elements in $M_{\mu}^{[a, b]}$ with respect to $\leq_{c x}$, that is, $\underline{X} \leq_{c x} U \leq_{c x} \bar{X}$ for all $U \in M_{\mu}^{[a, b]}$. The following lemma shows that such elements do exist and also characterizes them. For a real number $a$, let $\delta_{a}$ be the random variable with its whole mass at $a$, that is, $\operatorname{Pr}\left[\delta_{a}=a\right]=1$.

Lemma 2 : Let $\underline{X}=\delta_{\mu}$ and $\bar{X}=\theta \delta_{a}+(1-\theta) \delta_{b}$, where $\theta=(b$ $-\mu) /(b-a)$. Then $\underline{X} \leq_{c x} U \leq{ }_{c x} \bar{X}$ for all $U \in M_{\mu}^{[a, b]}$.

Proof : Let $f$ be a convex function. Then for any random variable $U \in M_{\mu}^{[a, b]}, \mathrm{E} f(\underline{X})=f(\mu)=f(\mathrm{E} U) \leq \operatorname{E} f(U)$ holds by Jensen's inequality. Hence $\underline{X} \leq{ }_{c x} U$. Now consider the remaining inequality. Note that $F_{\underline{X}}(t)=\theta$ for all $t \in[a, b)$ and $F_{\underline{X}}(b)=1$. If $F_{U}(a) \geq F_{\bar{X}}(a)$, then $F_{U}(t) \geq F_{\bar{X}}(t)$ for all $t \in[a, b]$. Hence the cut criterion is satisfied with $t_{0}=a$ and so $U \leq{ }_{c x} \bar{X}$. Now if $F_{U}(a)<F_{\bar{X}}(a)$, then since $F_{U}(b)=1$, there exists $t_{0} \in(a, b]$ which satisfies the cut criterion, which completes the proof.

By using the above lemma, we have the following :

$$
\begin{aligned}
& \Pi_{\bar{X}}(x) \leq \Pi_{U}(x) \leq \Pi_{\underline{X}}(x), \\
& \text { for all } x \geq 0 \text { and } U \in M_{\mu}^{[a, b]},
\end{aligned}
$$

which implies

$$
\Pi_{\bar{X}}^{*} \leq \Pi_{U}^{*} \leq \Pi_{\underline{X}}^{*} \text {, for all } U \in M_{\mu}^{[a, b]} .
$$

It can be shown that $\Pi_{\underline{X}}^{*}=r-c / \mu$ and $\Pi_{\bar{X}}^{*}=\max \left[\Pi_{\bar{X}}\right.$ $\left.(1 / a), \Pi_{\bar{X}}(1 / b)\right]$. Thus we get the following distributionfree bound on the maximum profit.

Proposition 5 : For all $U \in M_{\mu}^{[a, b]}, \max \left[\Pi_{\bar{X}}(1 / a), \Pi_{\bar{X}}(1 / b)\right]$ $\Pi_{U}^{*} \leq r-c / \mu$.

To get more insight, let us consider the special case of $a=0$ and $b=1$. In this case, it can be easily seen that $\Pi \frac{*}{X}=$ $\Pi_{\bar{X}}(1)=(r+p) \mu-(p+c)$. Hence we get the following result.

Corollary $1:$ For all $U \in M_{\mu}^{[0,1]},(r+p) \mu-(p+c) \leq \Pi_{U}^{*} \leq$ $r-c / \mu$.
Note that in Corollary 1, both the lower and the upper bounds are increasing in the mean yield.

\section{Concluding Remarks}

This note explores the stochastic ordering among the random yields and its implications on the maximum profit. The convex order plays an important role in comparing the maximum profit, especially with respect to the variance of the yield. When the random yield can be modeled as a usual parametric distribution, the variance reduction results in more profit. Also the mean-preserving transform supports the result. Hence in many cases, we can conclude that the variance reduction can contribute to improve the firm's profitability. The distribution-free bound given will be very helpful in estimating the profit when the random yield cannot be accurately characterized.

\section{References}

Anupindi, R. and Akella, R. (1993), Diversification under supply uncertainty, Management Sci., 29, 944-963.

Gerchak, Y. and Mossman, D. (1992), On the effect of demand randomness on inventories and costs, Operations Research, 40, 804-807.

Gerchak, Y. and Parlar, M. (1990), Yield variability, cost tradeoffs and diversification in the EOQ model, Naval Res. Logistics, 37, 341-354.

Gerchak, Y., Parlar, M., and Vickson, R. G. (1986), A single period production model with uncertain output and demand, Proc.25th IEEE Conf. on Decision and Control, Athens, Greece, 1733-1736.

Gupta, D. and Cooper, W. L. (2005), Stochastic comparisons in production yield management, Operations Research, 53, 377-384.

Henig, M. and Gerchak, Y. (1990), The structure of period review policies in the presence of variable yield, Operations Research, 38, 634643.

Keren, B. (2009), The single-period inventory problem : extension to random yield from the perspective of the supply chain, Omega, 37, 801810.

Khouja, M. (1999), The single-period newsvendor problem : literature review and suggestions for further research, Omega, 27, 537-253.

Muller, A. and Stoyan, D. (2002), Comparison Methods for Stochastic Models and Risks, John Wiley and Sons, Chichester, UK.

Park, K. and Lee, K. (2014), A Comprehensive analysis on the single-period inventory control problem under random yield and certain demand, Working Paper.

Shaked, M. and Shanthikumar, J. G. (1994), Stochastic Orders and Their Applications, Academic Press, New York.

Shih, W. (1980), Optimal inventory policies when stockouts result from defective products, Internat. J . Production Res., 18, 677-685.

Yano, C. A. and Lee, H. L. (1995), Lot sizing with random yields : A review, Operations Research, 43(2), 311-334. 